\title{
Quae nec pernumerare curiosi / Possint nec mala fascinare lingua:' Quantificazione numerica indeterminata in latino
}

Federica Fumante

(University of Rome "Tor Vergata")

\section{Quae nec pernumerare curiosi / Possint nec mala fascinare lingua: ${ }^{1}$ Indeterminate numerical quantification in Latin}

\begin{abstract}
According to their nature, the cardinal numerals define quantities and measures accurately. Nevertheless, in the conversational commonplaces of languages, the cardinal numerals can mean generic, indeterminate, and approximate quantities. The values expressed by these locutions are often situated on the two opposite poles of the numerical scale: they can signify a 'small', 'very small' amount or a 'great', 'enormous' sum. Similar expressions are used by almost every linguistic community. The numeral involved and the lexeme matched may change according to sociolinguistic variations: geographic (diatopic) differences, communicative contexts (diaphasic), and sociocultural stratifications (diastratic). Even in Latin, there are expressions of the same kind, typical of colloquial uses. For instance, 'one hundred' and 'one thousand' designate a large or a huge quantity: Da mi basia mille, deinde centum, Dein mille altera, dein secunda centum (Catull., carm. 5). Our analysis will focus on indeterminate quantifications in Latin locutions with numerals, starting from examples showed by Catullus' Liber.
\end{abstract}

\section{Keywords}

Latin; numerals; Catullus; indeterminacy; quantification; approximation

1 Catull. 7,11-12. 
All'atto della ricezione e/o della produzione del linguaggio numerico, il rigore di quest'ultimo impone l'eliminazione di qualsiasi elemento che trascini con sé soggettività e che, stimolando la situazione paratestuale, lasci spazio all'imprecisione. Tuttavia, nell'arco di ventiquattro ore, il parlante, indipendentemente dalla categoria diastratica di appartenenza, utilizza i numerali oltre che per calcoli, previsioni o bilanci economici, anche per raccontare la realtà che lo circonda, esprimendo all'interno degli enunciati quantità indeterminate di varia portata, talvolta infinitesimamente piccole o grandi.

L'atto di "contare" 2 - dal latino computare ${ }^{3}$ - con assolutezza, e quello di "raccontare", talora con ambiguità e imprecisione, possono configurarsi come operazioni sorelle (Bernardini \& De Mauro 2003). Da un punto di vista linguistico, ciò è segnalato da una serie di evidenze, in prima istanza dalla formazione del verbo "raccontare" per derivazione da "contare" intorno alla fine del XIII sec: ${ }^{4}$

1) "Storia è a raccontare le antiche cose state veramente, le quali furono dinnanzi a nostro tempo, e fuori di nostra memoria." (Tesoro di Brunetto Latini volgarizzato da Bono Giamboni)

Anche nel francese moderno, il latino computare è all'origine sia del lessema indicante l'operazione del calcolo sia di quello designante l'attività di parola (fr. compter "contare", conter "raccontare"). Questi esempi richiamano l'antichissima interrelazione tra pensiero scientifico e linguaggio che già il greco lògos racchiudeva in sé.

Oltre che linguistico, il nesso tra "contare" e "raccontare" è molto forte e significativo anche da un punto di vista culturale. Così Calvino:

"L'atteggiamento scientifico e quello poetico coincidono: entrambi sono atteggiamenti insieme di ricerca e di progettazione, di scoperta e di invenzione."5

I numerali appartengono, sotto aspetti diversi, al campo scientifico e umanistico, esattamente come al campo d'azione del quotidiano in cui, da una parte, rispettiamo il rigore dei numeri "contando", dall'altra, "raccontando", li usiamo in modo elastico e disinvolto.

All'inizio della sua attestazione linguistica, "contare" registra molteplici sfumature semantiche del "parlare", del "narrare" e del "dire" che convoglieranno poi in "raccontare": 2) Lo duca stette un poco a testa china; poi disse: "Mal contava la bisogna colui che i peccator di qua uncina" (Dante Alighieri, Inferno, Canto XXIII,140-141).

Ad avvenuta stabilizzazione semantica di "raccontare", "contare", tuttavia, continua a mantenere l'accezione di elencare - come se ad opera di un "resoconto" - una o più azioni, accadimenti o altro. Ad esempio nel linguaggio letterario:

3) Odi, Melisso: io vo' contarti un sogno di questa notte, che mi torna in mente in riveder la luna (Giacomo Leopardi, Canti, XXXVII).

4) 'Ntoni, quando la sera tornava a casa, non trovava altro che le donne, le quale mutavano la salamoia nei barilotti, e cianciavano in crocchio colle vicine, sedute sui sassi;

2 Pfister \& Schweickard (1984), De Mauro (2000).

3 Ernout \& Meillet (1932), De Vaan (2008).

4 Anche la forma inglese recounting "raccontare" è derivata a partire da counting "contare" (Meyer-Lübke 1972).

5 Calvino (1995: pp. 103-104). 
e intanto ingannavano il tempo a contare storie e indovinelli, buoni pei ragazzi, i quali stavano a sentire con tanto d'occhi intontiti dal sonno (Verga, I Malavoglia, XI).

Ma ancora oggi in alcuni dialetti, come nel napoletano:

5) Chitarra mia,

l'aggio vuluto bene e sulo tu

cunosce chisto ammore;

cumpagna mia,

venevo a cuntà a te 'ncopp' a sti ccorde

'e ppene 'e chisto core,

e mo ca essa ha pigliato n'ata via

che t'aggia cuntà cchiù chitarra mia.

(Antonio De Curtis e Michele Cozzoli, Chitarra mia)

I numerali assolvono moltissime funzioni: sono utilizzati nel calcolo, sono indicatori di una specifica numerosità, segnalano la posizione di un elemento all'interno di una serie ordinata; a ciò si aggiunge che il codice linguistico, tramite l'uso di modificazioni morfologiche, ordinamenti sintattici e usi allegorici, può attribuire ulteriori prerogative a questi lessemi. ${ }^{6}$ La nostra indagine prenderà in considerazione le occorrenze dei numerali con valore indeterminato a partire dal Liber di Catullo, verificando anche in quale misura questo tipo di espressioni ricorrano altrove all'interno del latino. Per focalizzare questa proprietà dei numerali, abbiamo analizzato le espressioni in prospettiva pragmatica con attenzione al contesto, all'interazione discorsiva, alle intenzioni del parlante, all'elemento sociale, culturale ed emotivo, espresso o implicito.

\section{L'imprecisione di un linguaggio preciso}

Quotidianamente utilizziamo scientificamente i numerali cardinali con precisione, tuttavia, spesso è impossibile o inutile essere precisi, per mancata conoscenza di dati definitivi e attendibili:

“[...] Contare i fili d'erba è inutile, non s'arriverà mai a saperne il numero. Un prato non ha confini netti, c’è un orlo dove l'erba cessa di crescere ma ancora qualche filo sparso ne spunta più in là, poi una zolla verde fitta, poi una striscia più rada: fanno ancora parte del prato o no? Altrove il sottobosco entra nel prato: non si può dire cos'è prato e cos'è cespuglio. Ma pure là dove non c'è che erba, non si sa mai a che punto si può smettere di contare: tra pianticella e pianticella c'è sempre un germoglio di fogliolina che affiora appena dalla terra e ha per radice un pelo bianco che quasi non si vede; un minuto fa si poteva trascurarla ma tra poco dovremo contare anche lei. Intanto altri due fili che poco fa sembravano appena un po' giallini ecco che ora sono definitivamente appassiti e sarebbero da cancellare dal conto. Poi ci sono le frazioni di fili d'erba, troncati a metà, o rasi al suolo, o lacerati lungo le nervature, le foglioline che hanno perso

6 Per un approfondimento sulla categoria linguistica dei numerali cfr. Holmer (1966), Hurford (1975), Corbett (1978), Pannain (2000). 
un lobo... I decimali sommati non fanno un numero intero, restano una minuta devastazione erbacea, in parte ancora vivente, in parte già poltiglia, alimento d'altre piante, humus..."

Altre volte si vuol fuggire dall'eccessiva specificazione o, ancora, si preferisce sfumare e attenuare la percezione del reale.

Nella prassi conversazionale utilizziamo, dunque, i numerali cardinali anche in espressioni che veicolano quantità generiche, indeterminate e approssimative. ${ }^{8}$

I quantitativi indicati da queste espressioni si collocano, spesso, alle due estremità opposte della scala numerica: designano cioè il 'poco' o 'pochissimo' e il 'molto' o 'moltissimo' ma possono anche, a seconda del contesto, assumere valori di polarità opposta. Le espressioni di questo tipo sono spesso fissate in locuzioni idiomatiche proprie di ciascuna comunità linguistica. La scelta, sia del numerale sia dell'elemento lessicale abbinato, è soggetta a variazioni sociolinguistiche, subordinate a differenze regionali (diatopiche), ai contesti comunicativi (diafasiche), alle stratificazioni socio-culturali (diastratiche). Anche il latino presenta usi di questo tipo, che potremmo definire iperbolici, appartenenti allo stile colloquiale e al linguaggio figurativo:

6) Catull. 5,7-9:

Da mi basia mille, deinde centum, dein mille altera, dein secunda centum, deinde usque altera mille, deinde centum

"Dammi mille baci, e poi cento, poi altri mille e altri cento, poi ancora altri mille e altri cento."

Nella locuzione, i numerali mille e centum designano una quantità elevata, quasi tendente all'infinito.

Nell'utilizzo approssimativo, ${ }^{9}$ al numerale più vicino al valore esatto rappresentato, è anteposto un approssimatore, spia del fatto che i dati numerici non sono forniti con precisione. ${ }^{10}$ Ad esempio:

ad:

7) Ter. Haut. 144-146:

Inscripsi ilico aedis mercede: quasi talenta ad quindecim coegi

"Subito ho messo in affitto la mia casa. Ne ho ricavato circa quindici talenti."

fere:

8) Ter. Hec. 822-824:

7 Calvino (1994: p. 30).

8 Cfr. McCarthy \& Ronald (2004), Lavric (2010), Voghera (2017).

9 Per approssimare, la lingua italiana utilizza indicatori di approssimazione, singolarmente o in combinazione tra loro, come: "circa", "quasi", "approssimativamente", ecc.; adopera anche risorse lessicali indicanti una quantità indeterminata vicina alla cardinalità esatta, ma non coincidente perfettamente con essa, ad esempio: "dozzina", "quindicina", "ventina”, "centinaio". Possiede, infine, dei lessemi che non rimandano a riferimenti numerici ma che quantificano genericamente, ad esempio: "pugno" e "pizzico" per una piccola quantità, "sacco" e "mucchio" per una ingente. Per esempi nell'utilizzo di questi ultimi in latino, si veda Bazzanella (2011b).

10 Per un approfondimento sull'approssimazione in latino si veda Bertocchi \& Orlandini \& Poccetti (2016). 
nam memini abhinc mensis decem fere ad me nocte prima confugere anhelantem domum sine comite, vini plenum, cum hoc anulo.

"Mi ricordo che circa dieci mesi fa Panfilo corse a rifugiarsi da me sul fare della notte, ansimante, senza scorta, ubriaco fradicio, e aveva questo anello."

\section{circiter:}

9) Caes. Gall. 1,26,5:

Ex eo proelio circiter milia hominum cxxx superfuerunt eaque tota nocte continenter ierunt: nulla partem noctis itinere intermisso in Lingonum die quarto pervenerunt, cum et propter vulnera militum et propter sepulturam occisorum nostri tridum morati eos sequi non potuissent

"I superstiti della battaglia, circa centrotrentamila uomini, si ritirarono, camminando initerrottamente tutta la notte e, senza mai fermarsi, nel quarto giorno arrivarono nelle terre dei Lingoni: i nostri, occupati per tre giorni a curare le ferite dei soldati e a seppellire i morti, non li poterono inseguire."

Lo stesso obiettivo può essere ottenuto anche coordinando con asindeto un numerale e il susseguente:

10) Ter. Eun. 331-333:

Illum liquet mihi deierare his mensibus sex septem prorsum non vidisse proxumis, nisi nunc quom minime vellem minimeque opu' fuit.

"Potrei tranquillamente giurare di non averlo mai visto negli ultimi sei sette mesi, tranne adesso, quando era l'ultima cosa al mondo che desideravo e di cui avevo bisogno."

Ancora, è possibile approssimare tracciando un limite numerico di confine in cui è racchiuso l'intervallo quantitativo.

Limite numerico inferiore: non minus

Limite numerico superiore: non plus

11) Vitr. 5,7,2:

Eius logei altitudo non minus debet esse pedum $x$, non plus duodecim.

"L’altezza del palcoscenico non dev'essere inferiore ai dieci piedi né superare i dodici."

Altrove, per delimitare l'area intorno a cui si aggira la cifra esatta, è segnalato soltanto il limite numerico superiore.

12) Nep. Thras. 2,1:

Hic enim cum Phylen confugisset, quod est castellum in Attica munitissimum, non plus habuit secum triginta de suis.

"Quando infatti si rifugiò a File, che è una fortezza dell'Attica molto ben difesa, aveva con sé non più di trenta sostenitori."

Tale indicatore di limite superiore, a seconda del contesto comunicativo, può essere utilizzato quasi esclusivamente con intento di precisione; ciò accade talvolta nel dialogo. Infatti, nella prassi colloquiale, essendo più inclini all'uso indeterminato e approssimativo che a quello cardinale, quando vogliamo essere interpretati alla lettera, utilizziamo indicatori linguistici di precisione, come ad esempio nell'italiano: "Me ne dia un etto, 
non di più" singolarmente o in combinazione: "Me ne dia appena/giusto un etto, non di più". Anche il latino conosce usi di questo tipo che informano sulla soglia quantitativa stabilita che non è possibile superare:

13) Caecil. com. 51-52:

uelim paulisper te opperíri.

Quantisper?

Non plus tríduum.

"Vorrei che tu aspettassi un po'.

Cosa intendi per un po'?

Non più di tre giorni."

\section{Indeterminatezza numerica}

Analizzeremo di seguito un corpus che raccoglie usi "convenzionalizzati" (Bazzanella 2011a) di numerali cardinali, adoperati volontariamente con valore indeterminato; tale uso è desumibile dal contesto in cui è inserito il sintagma frastico contenente il numerale in questione, il cui valore non è da accogliere letteralmente ma da interpretare.

\section{1 unus, una, unum}

14) Catull. 5,1-3:

Vivamus, mea Lesbia, atque amemus

Rumoresque senum severiorum

Omnes unius aestimemus assis!

"Viviamo, Lesbia mia, ed amiamoci,

e i brontolii dei vecchi austeri

valutiamoli, tutti insieme, un sol soldo.”

Unus, una, unum, partendo dal suo valore cardinale indicante l'unità, può decrescere fino a identificare lo "zero". Nel presente contesto, infatti, il numerale "uno" ha valore di 'minimizer'; per il poeta, dunque, non bisogna attribuire alcuna stima al chiacchiericcio di chi, invidioso della passione amorosa sua e di Lesbia, va blaterando.

15) Catull. 67,15-16:

Non istuc satis est uno te dicere verbo,

Sed facere ut quiuis sentiat et videat.

"Sì, ma non te la cavi con queste sole parole,

devi far sì che chiunque possa sentire e vedere"

Ugualmente unus può, scardinandosi dal valore letterale, significare pochezza. Uno verbo nell'esempio indicherebbe "poche parole", quello che in italiano, utilizzando un'espressione con un numerale dal valore indeterminato, si tradurrebbe in 'due parole'; in un'espressione dallo stesso significato, il numerale adottato può, infatti, diversificarsi al variare della lingua presa in considerazione. ${ }^{11}$

11 Strudsholm (2011). 


\section{2 duo, duae, duo}

C. Bazzanella (2011a: p. 21) afferma:

"L'uso, convenzionalizzato in italiano, del numero due per indicare una quantità piccola in modo cortese è talmente frequente nella vita di tutti i giorni che in genere non lo notiamo, così come non notiamo usi simili di numeri cardinali sia per diminuire che per aumentare, con un valore paradossalmente approssimato".

Tale uso di 'due' per veicolare una quantità minima indeterminata si trova ugualmente espresso in latino. "Due", nell'esempio indica "pochi, pochissimi" non letteralmente "due":

16) Pers. 1,1-3:

O curas hominum! o quantum est in rebus inane!

'quis leget haec?' min tu istud ais? nemo hercule. 'nemo?'

vel duo vel nemo.

"O passioni umane, o quanta vanità nelle cose!

'Già; ma chi vuoi che legga roba di questo genere?'

A me lo chiedi? Nessuno, per Ercole.

'Nessuno?'

O due o nessuno."

\section{3 tres, tria}

Il senso di quantità minima può essere espresso anche dal numerale 'tre':

17) Ter. Phorm. 636-639:

Ge: Nam sat scio, si tu aliquam partem aequi bonique dixeris ut ěst ille bonus vir, tria non commutabitis verba hodie inter vos.

"Geta: è un brav'uomo, lo conosco bene: se fai una proposta ragionevole, vi metterete d'accordo subito, in due parole."

In questo esempio tria verba è similare al senso del numerale "uno" (uno verbo) dell'esempio precedente (esempio 15) tratto dal Liber di Catullo; sebbene il numerale associato a verbum sia diverso, entrambi tendono verso il limite numerico inferiore, venendo così a significare una piccola quantità, con funzione di quantificatori minimali (a volte deprezzativi).

\section{5 quinque}

Dal "cinque" in poi, i numerali iniziano a orientarsi in direzione del polo numerico positivo, passando ad indicare la moltitudine generica:

18) Pers. 4,39-41:

Quinque palaestritae licet haec plantaria vellant

elixasque nates labefactent forcipe adunca, non tamen ista filix ullo mansuescit aratro.

"Anche ammesso che cinque palestriti strappino questa selva e strazino le flosce natiche con pinzette adunche, si può star certi che non c'è aratro 
che riesca a domare codesta erbaccia."

Presentando, tuttavia, ancora qualche oscillazione: il "cinque", infatti, può anche esprimere un valore esiguo tendente alla polarità opposta negativa:

19) Hor. sat. 1,3,15-17:

Deciens centena dedisses huic parco, paucis contento, quinque diebus nil erat in loculis.

"Potevi dargli un milione di sesterzi, a quest'uomo parco, contento di poco: in cinque giorni non gli restava niente in tasca."

\section{7 septem}

Il "sette" assume negli esempi riportati valore cardinale. Il numerale trova scarso impiego nelle approssimazioni indeterminate, probabilmente a causa delle sue forti caratterizzazioni storico-culturali, si pensi ai sette colli:

20) Stat. silv. 2,3,20-22:

Ecce citatos advertit Diana gradus, dum per iuga septem errat Aventinaeque legit vestigia cervae.

"Ma Diana, errante peri sette colli, nell'inseguire le orme d'una cerva dell'Aventino, si dirige là a rapidi passi."

ma anche alle sette portate della cena vera e propria, la mensa prima: ${ }^{12}$

21) Ivv. 1,94-95:

Quis totidem erexit villas, quis fercula septem secreto cenauit avus?

"E chi ha mai costruito tante ville, e chi mai, tra i nostri antenati, cenava con sette portate sol per sé ?"

\section{8 octo: "molti"}

22) Plaut. Mil. 826-832:

LVC. Quid vis?

PAL. Qui lubitum est illi condormiscere?

LVC. Oculis opinor.

PAL. Non te istuc rogito, scelus.

Procede huc. Periisti iam, nisi verum scio.

Prompsisti tu illi vinum?

LVC. Non prompsi.

PAL. Negas?

LVC. Nego hercle vero, nam ille me vetuit dicere;

neque equidem heminas octo exprompsi in urceum

neque illic calidum éxbibit in prandium.

"LVC. Be'?

PAL. Come gli è venuta la voglia di dormire?

LVC. Come? Con gli occhi credo.

12 Nell'esempio, il "sette" potrebbe, al più, esprimere "la totalità", la completezza. Anche al di fuori della latinità, il numerale assume moltissimi valori simbolici, spesso referenziali di enti astratti; si pensi ai "sette giorni della settimana", ai "sette vizi capitali", alle "sette note musicali". 
PAL. Non è questo che ti chiedevo, mascalzone.

Vieni qui. Sei bell'e morto se non mi dici la verità. Sei tu che gli hai versato il vino?

LVC. No.

PAL. Dici di no?

LVC. Dico di no, per Ercole, perché lui mi ha proibito di parlare: di conseguenza non è vero che ho spillato nel suo orcio circa otto emine < circa quattro litri > di vino e che lui se li è bevuti belli caldi a pranzo.”

9 novem: "molti"

23) Catull. 32,4-8:

Et si iusseris, illud adiuvato,

Ne quis liminis obseret tabellam,

Neu tibi lubeat foras abire

sed domi maneas paresque nobis

novem continuas fututiones.

"Se sei d'accordo, devi anche

far sì che nessuno chiuda il chiavistello

e non farti venire la voglia di uscire:

resta a casa e preparami

nove scopate di fila"

\section{0 decem}

Generalmente, indica una quantità indefinita tendente alla moltitudine:

24) Plaut. Mil. 354-356:

Pal. Praecépta facito ut mémineris. Phil. Totiéns monere mírumst. Pal. At metuo ut satis sis subdola. Phil. Cedo vel decem, edocebo minime malas ut sint malae, mihi solae quod superfit.

"Pal: Ricordati bene quel che ti ho detto. Phil: Non c'è proprio bisogno di ripeterlo tante volte! Pal: Ho paura che tu non sia abbastanza furba. Phil: Figurati! Potrei istruire dieci ingenue e farle diventare perverse solo con quel tanto di furbizia che mi avanza!"

Tuttavia, decem in associazione a vix indica un quantitativo scarso:

25) Ter. Andr. 452-453:

Da. Te. "vix" inquit "drachumis est opsonatum decem: non filio videtur uxorem dare".

"Davo: Tu. 'Per il banchetto' dice 'avrà speso sì e no dieci dracme, si direbbe che non sia suo figlio a sposarsi'."

26) Ter. Haut. 908-909:

Ch. fili est amica Bacchi': Menedeme, occidi. Me. quăm ŏb rem? Ch. decem dierum vix mi est familia.

"Ch: Bacchide è l'amica di mio figlio: Menedemo, sono spacciato!

Me: Perché mai?

Ch: La mia casa avrà vita breve: dieci giorni al massimo!" 
Vix è un avverbio scalare, indica sulla scala positiva una quantità molto piccola, vicina al polo negativo:

27) Cic. Lael. 15:

Itaque non tam ista me sapientiae, quam modo Fannius commemoravit, fama delectat, falsa praesertim, quam quod amicitiae nostrae memoriam spero sempiternam fore, idque eo mihi magis est cordi, quod ex omnibus saeculis vix tria aut quattuor nominantur paria amicorum;

"Pertanto mi fa piacere non tanto questa fama di saggezza che ha ricordato Fannio, oltretutto falsa, quanto il fatto che spero che la memoria della nostra amicizia durerà in eterno; e ciò mi sta maggiormente a cuore, poiché, di tutti i secoli passati, a stento vengono ricordate tre o quattro coppie di amici."

Tria aut quattuor esprimono, figurativamente, un numero parco di amici. Viceversa:

28) Lucr. 4,304-305:

Fit quoque de speculo in speculum ut tradatur imago, quinque etiam <aut> sex ut fieri simulacra suërint. "Anche accade che da specchio a specchio si trasmetta l'immagine, sì che sogliono prodursi addirittura cinque <o> sei immagini."

"Cinque o sei" identificano un numero notevole di immagini. Etiam "anche" conferisce all'approssimazione un valore generico tendente alla molteplicità. A differenza di quoque che indica uguaglianza, etiam può avere un uso scalare tendente verso l'alto. ${ }^{13}$ Per ciò che concerne l'espressione delle centinaia, i numerali esprimono la moltitudine indeterminata non ancora tendente all'infinitezza innumerabile.

\section{0 centum}

Esempi di espressioni in cui il numerale "cento" è utilizzato con valore indeterminato sono il già citato carme di Catullo (esempio 6) ma anche la prima e la quinta Satira di Persio:

29) Pers. 5,1-4:

Vatibus hic mos est, centum sibi poscere voces,

Centum ora et linguas optare in carmina centum,

fabula seu maesto ponatur hianda tragoedo, vulnera seu Parthi ducentis ab inguine ferrum.

"È abitudine dei poeti invocare di per sé cento voci,

di augurarsi per i loro carmi cento bocche e cento lingue,

sia che si tratti di una tragedia che l'attore sonoramente

declamerà con faccia lugubre, sia che si debbano cantare

le ferite del Parto che si strappa il ferro dall'inguine."

30) Pers. 1,29-30:

Ten cirratorum centum dictata fuisse pro nihilo pendes?

"E ti par poco essere dettato a cento ricciutelli?"

13 A tal proposito si veda Orlandini (2001). 
Nell'esempio 29, il numerale "cento" con valore indeterminato è presente ben tre volte soltanto nei primi due versi. Il parallelo con l'esempio di Catullo consiste anche nell'iterazione del numerale ripetuto a distanza.

Verso le 'centinaia', i quantitativi elevati espressi diventano quasi innumerabili:

300 trecenti, ae, a

31) Catull. 11,17-20:

cum suis vivat valeatque moechis,

quos simul complexa tenet trecentos,

nullum amans vere, sed identidem omnium

ilia rumpens

"Viva e se la spassi con i suoi amanti,

che, trecento per volta, si stringe fra le braccia,

senza amarne nessuno veramente, ma sfiancando

a tutti e trecento le reni"

32) Catull. 12,10-11:

quare aut hendecasyllabos trecentos

exspecta, aut mihi linteum remitte

"Concludendo: o ti aspetti trecento endecasillabi

o a me tu restituisci il fazzoletto"

L'indeterminatezza tendente al limite superiore positivo si corrobora in presenza dell'associazione di due numerali esprimenti cifre elevate:

\section{0-200 centum an ducenti}

33) Catull. 37,6-8:

An, continenter quod sedetis insulsi

centum an ducenti non putatis ausurum

me una ducentos irrumare sessores?

"Perché sedete là in cento o duecento come stupidi,

vi siete fatta l'idea che io non osi fottervi

in una volta tutti e duecento?"

\section{0-300 ducenties aut trecenties}

34) Catull. 29,13-14:

ut ista vestra diffututa mentula

ducenties comesset aut trecenties? ${ }^{14}$

"perchè venti o trenta milioni si pappasse

questa vostra minchia smidollata?"

14 Nell'esempio i numerali sottintendono 'centena milia sestertium'. 
Nei due esempi, i numerali potrebbero sostituirsi con altri indicanti cifre elevate, esprimendo la stessa idea di moltitudine indeterminata. Il criterio della commutazione permette, infatti, di riconoscere l'impiego indeterminato da quello cardinale (Lavric 2007): nella designazione indeterminata i numeri risultano sostituibili tra loro purché il senso ultimo del sintagma frastico contenente il numerale risulti immutato. Si noterà come, a volte, la scelta ricada su un determinato numerale per ragioni di metrica o nel rispetto della tradizione culturale e/o linguistica.

Due numerali in correlazione possono esprimere la moltitudine indefinita quando il secondo numerale supera il primo in valore; diversamente, la specificazione numerica manifesta un intento di precisione.

\section{0 mille}

"Mille" è il numerale più frequentemente utilizzato per indicare una quantità indeterminata elevata innumerabile, si pensi all'italiano "grazie mille / mille grazie", ma anche ad altre espressioni quali: "mille volte" (eng. "a thousand times") ancora: "avere mille pensieri / idee per la testa" (eng. "a thousand thoughts"); ad espressioni proverbiali come: "il silenzio vale più di mille parole" e metaforiche: "farsi di mille colori". ${ }^{15}$ Tale utilizzo era già presente nel latino e forse affonda le sue radici ancora più indietro; ne è l'esemplificazione più famosa l'emistichio Catulliano Da mi basia mille (esempio 6). Altri utilizzi indeterminati ricorrono in Stazio, nell'Egloga ad uxorem:

35) Stat. silv. 3,5,6-10:

Et si egomet patrio de litore raptus

quattuor emeritis per bella, per aequora lustris

errarem, tu mille procos intacta fugares,

non imperfectas commenta retexere telas,

sed sine fraude palam thalamosque armata negasses.

"Anche se strappato dai patri lidi io andassi errando

per ben quattro lustri tra guerre e mari, tu metteresti in fuga

mille pretendenti, senza lasciarti toccare, né fingere

di tessere tele disfatte, ma palesemente e senza inganno,

e con le armi in pugno negheresti l'acceso alle tue stanze."

in Persio:

36) Pers. 5,52:

Mille hominum species et rerum discolor usus

"l'umanità offre mille aspetti diversi e svariata condotta"

e nel Bellum Civile di Lucano:

37) Luc. 1,299-302:

"Bellorum o socii, qui mille pericula Martis

mecum" ait "experti decimo iam vinctis anno,

15 Oltre che dal lessico conversazionale, emergono dall'italiano, ma anche dalle altre lingue, riferimenti dalla letteratura e dalla poesia, dalla musica, dalla pubblicità, dal lessico televisivo e giornalistico. 
hoc cruor Arctois meruit diffusus in arvis

vulneraque et mortes hiemesque sub alpibus actae?"

"Compagni di guerra" disse "che esperti con me dei pericoli

innumerevoli di Marte vincete ormai da dieci anni,

questo ha meritato il sangue sparso nelle terre nordiche,

e le ferite, e le morti, e gli inverni trascorsi ai piedi delle Alpi?"

Anche i derivati da mille sono soggetti allo stesso uso:

Millies

38) Catull. 35,7-10:

Quare, si sapiet, viam vorabit

quamvis candida millies puella

euntem revocet manusque collo

ambas iniciens roget morari.

"Se ha cervello,

divorerà la strada anche se mille volte

lo richiamerà, mentre già è in via, la sua bellissima

ragazza e, gettandogli al collo ambedue

le braccia, lo implorerà di restare"

\section{Milia}

39) Catull. 61,199-203:

Ille pulveris Africi

siderumque micantium

subducat numerum prius,

qui vestri numerare vult

multa milia ludi

"Conti prima i granelli di polvere

nel deserto africano,

o le stele lucenti nel cielo

chi vuole contare

i vostri infiniti giochi amorosi”

Milia può accompagnarsi ad altri numerali che fungono da moltiplicativi enfatizzando la quantità massima incalcolabile:

40) Catull. 22,4-8:

Puto esse ego illi milia aut decem aut plura

perscripta, nec sit ut fit in palimpseston

relata: cartae regiae, novi libri,

novi umbilici, lora rubra, membranae,

derecta plumbo et pumice omnia aequata.

"Ne avrà scritti già diecimila

$\mathrm{o}$ anche più e non, come tutti, su un palinsesto, 
no, su carta di prima qualità, rotoli

nuovi, bastoncini nuovi, nastri rossi, astucci

di pergamena, tutto tracciato a piombo e tirato a lucido."

\section{$\mathbf{3 0 0 . 0 0 0}$}

41) Catull. 48,2-3:

Si quis me sinat usque basiare usque ad milia basiem trecenta

"se me li lasciassero baciare sempre, continuerei

a baciarli fino a un milione di volte"

42) Catull. 9,1-4:

Verani, omnibus e meis amicis

antistans mihi milibus trecentis

venistine domum ad tuos penates

fratresque unanimos anumque matrem?

"Veranio, che superi tutti i miei amici,

per me, di mille miglia,

finalmente sei tornato a casa,

dai fratelli affezionati, dalla vecchia mamma?"

In conclusione, usi indeterminati dei numerali sono frequenti in latino come anche in altre lingue antiche e moderne. Catullo è, tra i poeti latini, l'autore che meglio si fa portavoce delle quantificazioni numeriche indeterminate: nel trascrivere "l'emozione infinita", ${ }^{16}$ inesprimibile e incalcolabile, fa un grande e vario uso dei numerali con valore indefinito, offrendo così un ampio panorama a cui guardare. L'intervallo quantitativo che queste espressioni designano può orientarsi verso il polo numerico positivo o negativo a seconda del contesto comunicativo. Questa proprietà dei numerali potrebbe risultare scontata o banale in quanto naturale e universalmente insita negli individui appartenenti a comunità linguistiche con un avanzato dominio matematico $;{ }^{17}$ talvolta, però è proprio l'ovvietà a richiedere maggiori spiegazioni, e i numerali, nei loro usi quotidiani, scientifici e precisi, ma anche creativi e paradossali, offrono diversi motivi di interesse e riflessione linguistica in qualsivoglia cultura.

16 L'espressione è presa in prestito da Paduano (2016).

17 Lo sviluppo di una consapevolezza numerica sempre più elaborata è la risposta ad un bisogno puramente pratico, che scaturisce dalla necessità di comunicare (linguisticamente o meno) informazioni numeriche; i sistemi di numerazione, risultano, infatti, del tutto assenti o quasi in comunità linguistiche in cui lo sviluppo più articolato del 'senso numerico' non è un'esigenza contingente ai fini della sopravvivenza e della comunicazione quotidiana, ossia non è richiesto dal contesto. Ne costituisce un esempio la popolazione amazzonica dei Pirahã che utilizza un sistema di numerazione del tipo 'uno'/ 'due'/ 'molti' (Crevatin 1995). 


\section{Bibliografia}

Bazzanella, C. (2011a). Numeri per parlare. Da «quattro chiacchere» a «grazie mille». Bari: Laterza.

Bazzanella, C. (2011b). Tria uerba. Numeri e approssimazione in latino. In A. Balbo, F. Bessone, \& E. Malaspina (Eds.), Tanti affetti in tal momento. Studi in onore di Giovanna Garbarino (pp. 437-449). Alessandria: Edizioni dell'Orso.

Bernardini, C., \& De Mauro, T. (2003). Contare e raccontare: dialogo sulle due culture. Roma: GLF editori Laterza.

Bertocchi, A., Orlandini, A., \& Poccetti, P. (2016). Structures de l'approximation en latin. In H. B.-Z. Shyldkrot, S. Adler, \& M. Asnès (Eds.), Nouveaux regards sur l'approximation et la précision (pp. 20-33). Paris: Editions Honoré Champion.

Calvino, I. (1994). Palomar. Milano: Mondadori.

Calvino, I. (1995). Una pietra sopra. Discorsi di letteratura e società. Milano: Mondadori.

Corbett, G. (1978). Universals in the syntax of cardinal numerals. Lingua, 46, 355-368.

Crevatin, F. (1995). Numeri tra lingua, cognizione e cultura. Annali del Dipartimento di Studi del Mondo Classico e del Mediterraneo Antico Sezione Linguistica, 27, 107-120.

De Mauro, T. (2000). Grande dizionario italiano dell'uso. Torino: UTET.

De Vaan, M. (2008). Etymological Dictionary of Latin and the Other Italic Languages. Leiden: Brill.

Ernout, A., \& Meillet, A. (1932). Dictionnaire étymologique de la langue latine. Paris: Klincksieck.

Holmer, N. M. (1966). The semantics of numerals. In Årsbok utgiven av seminarierna för slaviska språk vid Lunds Universitet 1963-64 (pp. 5-22). Lund: Slaviska Institutet.

Hurford, J. R. (1975). The Linguistic Theory of Numerals. Cambridge: Cambridge University Press.

Lavric, E. (2007). Les numéraux approximatifs, ou: comment se fait-il que sept minutes soient toujours exactement sept minutes, mais que cinq minutes puissent parfois être beaucoup plus? In D. Trotter (Ed.), Actes du XXIVe Congrès International de Linguistique et de Philologie Romanes, Aberystwyth 2004 (Vol. 4; pp. 139-153). Tübingen: Niemeyer.

Lavric, E. (2010). Hyperbolic Approximative Numerals in Cross-Cultural Comparison. In G. Kaltenböck, W. Mihatsch, \& S. Schneider (Eds.), New Approaches to Hedging (pp. 123-164). Bingley: Emerald Group Publishing Limited.

McCarthy, M., \& Ronald, C. (2004). «There's millions of them»: hyperbole in everyday conversation. Journal of Pragmatics, 36(2), 149-184.

Meyer-Lübke, W. (1972). Romanisches etymologisches Wörterbuch. Heidelberg: Winter.

Orlandini, A. (2001). Négation et argumentation en latin. Grammaire Fondamentale du Latin. Tome VIII. Louvain-Paris: Peeters.

Paduano, G. (Transl.). (2016). Gaio Valerio Catullo: Le poesie. Torino: Einaudi.

Pannain, R. (2000). Numerali ed istanze di numerazione: note per un progetto di tipologia areale dei numerali. Annali del Dipartimento di Studi del Mondo Classico e del Mediterraneo Antico Sezione Linguistica, 22, 63-103.

Pfister, M., \& Schweickard, W. (1984). LEI Lessico etimologico italiano. Wiesbaden: L. Reichert.

Strudsholm, E. (2011). Numeri e numbers nelle espressioni idiomatiche da lingua a lingua. Lingua Italiana [retrieved 8.8.2019 from http://www.treccani.it/magazine/lingua_italiana/speciali/ Numeri/Strudsholm.html]. 
Voghera, M. (2017). Costruzioni di piccoli numeri: la vaghezza intenzionale in funzione. In O.-D. Balaş et al. (Eds.), L'expression de l'imprécision dans les langues romanes (pp. 162-175). Bucureşti: Ars Docendi.

Federica Fumante / federica.fumante@yahoo.it

School of Humanities and Philosophy

University of Rome "Tor Vergata"

Macroarea di Lettere e Filosofia, Via Columbia 1, 00133 Roma, Italy 